\title{
Interplay of Magnetic and Superconducting Proximity Effects in FSF Trilayers
}

\author{
Tomas Löfwander, Thierry Champel, Johannes Durst, and Matthias Eschrig \\ Institut für Theoretische Festkörperphysik, Universität Karlsruhe, 76128 Karlsruhe, Germany
}

(Dated: July 16, 2005)

\begin{abstract}
We present theoretical results on the interplay of magnetic and superconducting orders in diffusive ferromagnet-superconductor-ferromagnet trilayers. The induced triplet superconducting correlations throughout the trilayer lead to an induced spin magnetization. We include self-consistency of the order parameter in the superconducting layer at arbitrary temperatures, arbitrary interface transparency, and any relative orientation of the exchange fields in the two ferromagnets. We propose to use the torque on the trilayer in an external magnetic field as a probe of the presence of triplet correlations in the superconducting phase.
\end{abstract}

The importance of triplet pairing correlations in the interface region between a singlet superconductor and a ferromagnet recently became the focus of research in the field of spintronics [1, 2, 3, 4]. In contrast to clean triplet $p$-wave superconductors and superfluids, for diffusive materials $p$-wave correlations are suppressed and triplet correlations have $s$-wave orbital symmetry, but are odd in frequency [1]. In the case of a homogeneous magnetization of the ferromagnet, the spin projection of the triplet correlations on the quantization axis of the exchange field is zero. If, on the other hand, the distribution of the exchange field in the ferromagnet is inhomogeneous in space, then under suitable conditions [5] also triplet correlations with non-zero spin projection (equal spin pairs) are induced [1, 2, 3]. Triplet pairing correlations induce in turn a spin magnetization both in the ferromagnet and in the superconductor [ 6, , 7].

An important question is how to experimentally find good fingerprints of the triplet superconducting correlations. Theoretical work has been focused on calculations of $T_{c}[5,[8,[9]$, the local density of states (LDOS) [4, 10, 11], or to search for unconventional Josephson couplings [3, 12]. Experimentally, no smoking gun has been found although recently magnetization changes were observed through neutron reflectometry on multilayers of $\mathrm{YBa}_{2} \mathrm{Cu}_{3} \mathrm{O}_{7}$ and $\mathrm{La}_{2 / 3} \mathrm{Ca}_{1 / 3} \mathrm{MnO}_{3}$ [13]. Various other properties of FS heterostructures that could be influenced by triplet superconducting correlations have been measured, as for example a negative Josephson coupling $(\pi$ junctions) 14, and LDOS modulations 15.

In the following we present results for the induced triplet correlations and corresponding changes of the magnetization in a ferromagnet-superconductorferromagnet (FSF) trilayer with arbitrary misalignment of the exchange fields in the two $\mathrm{F}$ layers. We put forward signatures of triplet correlations that can be measured experimentally.

The FSF trilayer we consider is sketched in Fig. 1 We denote the layer thicknesses by $d_{F 1}, d_{S}$, and $d_{F 2}$ respectively. The $x$-axis is directed perpendicular to the layer interfaces with the origin at the center of the superconductor. The $z$-axis is aligned with the exchange field $\vec{J}_{1}$ in the left ferromagnet. The angle between the exchange fields $\overrightarrow{J_{1}}$ and $\overrightarrow{J_{2}}$ is denoted $\theta_{J}$. We also assume translational invariance in the $y$ - $z$-plane.

We use the quasiclassical theory of superconductivity for diffusive systems 16 that is formulated in terms of momentum averaged Green functions. The Green function for the trilayer, $\hat{g}\left(x, \epsilon_{n}\right)$, depends on the $x$-coordinate and on the Matsubara frequency $\epsilon_{n}=(2 n+1) \pi T$ ( $n$ integer, $T$ temperature). In standard notation (see e.g. $[5,17])$ the Green function is a $4 \times 4$ matrix in combined Nambu-Gor'kov (electron-hole) and spin space

$$
\hat{g}=\left(\begin{array}{cc}
g_{s}+\vec{g}_{t} \cdot \vec{\sigma} & \left(f_{s}+\vec{f}_{t} \cdot \vec{\sigma}\right) i \sigma_{y} \\
\left(f_{s}^{*}+\vec{f}_{t}^{*} \cdot \vec{\sigma}^{*}\right) i \sigma_{y} & g_{s}^{*}+\vec{g}_{t}^{*} \cdot \vec{\sigma}^{*}
\end{array}\right),
$$

where $f_{s}$ and $\vec{f}_{t}$ are singlet and triplet pairing amplitudes, $g_{s}$ and $\vec{g}_{t}$ are spin scalar and spin vector parts of the diagonal Green function, and the vector $\vec{\sigma}=\left(\sigma_{x}, \sigma_{y}, \sigma_{z}\right)$ is composed of Pauli spin matrices. The Green function satisfy a Usadel-type equation [16, 18]

$$
\left[i \epsilon_{n} \hat{\tau}_{3}-\hat{\Delta}-\vec{J} \cdot \hat{\sigma}, \hat{g}\right]+\frac{D}{\pi} \partial_{x}\left(\hat{g} \partial_{x} \hat{g}\right)=\hat{0}
$$

where $D$ denotes the diffusion constant (equal to $D_{F}$ in F and to $D_{S}$ in $\left.\mathrm{S}\right),[\hat{a}, \hat{b}]=\hat{a} \hat{b}-\hat{b} \hat{a}$, and $\hat{\sigma}=\operatorname{diag}\left(\vec{\sigma}, \vec{\sigma}^{*}\right)$. The quantities $\hat{\tau}_{1}, \hat{\tau}_{2}, \hat{\tau}_{3}$ and $\hat{1}$ denote the Pauli matrices and the unit matrix in electron-hole space. Eq. (2) is supplemented by a normalization condition $\hat{g}^{2}=-\pi^{2} \hat{1}$.
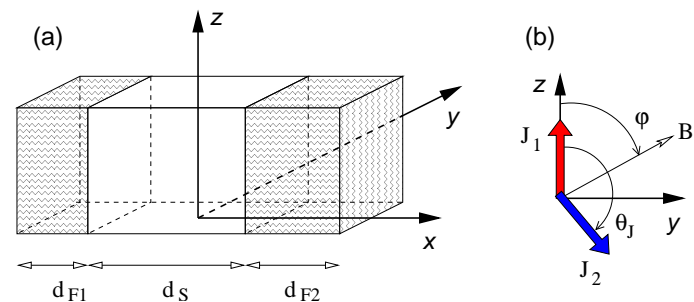

FIG. 1: The trilayer consists of a superconductor of thickness $d_{S}$ and two ferromagnets of thicknesses $d_{F 1}$ and $d_{F 2}$. The exchange fields of the ferromagnets, $\vec{J}_{1}$ and $\overrightarrow{J_{2}}$, are confined to the $y$-z-plane, but are misaligned by an angle $\theta_{J}$. For torque measurements, a small magnetic field is applied in the $y$-z-plane at an angle $\varphi$. 
The superconducting singlet order-parameter $\hat{\Delta}$ is determined by the pairing interaction, which we assume to be zero in the ferromagnetic parts of the trilayer. By a proper gauge transformation, $\hat{\Delta}$ can be chosen real, $\hat{\Delta}=\Delta i \sigma_{y} \hat{\tau}_{1}$, where $\Delta$ satisfies the gap equation

$$
\Delta(x) \ln \left(\frac{T}{T_{c 0}}\right)=\pi T \sum_{n}\left[\frac{f_{s}\left(x, \epsilon_{n}\right)}{\pi}-\frac{\Delta(x)}{\epsilon_{n}}\right] .
$$

The pairing interaction and the frequency-sum cut-off have been eliminated as usual in favor of the bulk superconductor critical temperature $T_{c 0}$. The trilayer critical temperature is lower $T_{c}<T_{c 0}$, but we use $T_{c 0}$ as a parameter independent energy scale in our problem.

The interface boundary conditions have been formulated by Nazarov [19] and consist of two equations. The first is the condition of current conservation through the interface $\sigma_{F} \hat{g}_{F} \partial_{x} \hat{g}_{F}=\sigma_{S} \hat{g}_{S} \partial_{x} \hat{g}_{S}$, where $\hat{g}_{F}$ and $\hat{g}_{S}$ denote the values of the Green function at the $\mathrm{F}$ and $\mathrm{S}$ sides of the interface, respectively. The normal state conductivities on either side are related to the corresponding diffusion constants $D$ and densities of states at the Fermi level $\mathcal{N}_{f}$ by $\sigma=2 \mathcal{N}_{f} e^{2} D$, where $e$ is the electron charge. Note that $\mathcal{N}_{f}$ in the up and down spin bands are to quasiclassical accuracy equal in the weak ferromagnet regime $T_{c}<|\vec{J}| \ll E_{f}$, where $E_{f}$ is the Fermi energy. The second boundary condition is written as [20]

$$
\sigma_{F} \hat{g}_{F} \partial_{x} \hat{g}_{F}= \pm \frac{1}{\mathcal{A} R_{b}} \frac{2 \pi^{2}\left[\hat{g}_{F}, \hat{g}_{S}\right]}{2 \pi^{2}(2-\mathcal{T})-\mathcal{T}\left\{\hat{g}_{F}, \hat{g}_{S}\right\}},
$$

where $R_{b}$ is the boundary resistance, $\mathcal{A}$ is the junction area, and $\mathcal{T}$ is the junction transparency. The sign $+(-)$ refers to the left (right) interface. For simplicity we use only two parameters: $\mathcal{T}$ and $r_{b}=\mathcal{A} R_{b} \sigma_{F} /\left(2 \pi^{2} \xi_{S}\right)$, where $\xi_{S}=\sqrt{D_{S} / 2 \pi T_{c 0}}$ is the coherence length in S. At the outer $\mathrm{F}$ surfaces the boundary conditions are $\partial_{x} \hat{g}_{F}=0$.

We have solved Eqs. (2)-(4) self-consistently with the help of a parametrization of the Usadel Green function in terms of Riccati amplitudes, as described in Ref. 21]. This method allows us to treat spatially inhomogeneous (including non-collinear) spin magnetizations.

The magnetic order (exchange fields $\vec{J}_{1}, \vec{J}_{2}$ ) and the superconducting order $(\Delta)$ are spatially separated. However, due to quantum mechanical leakage of the superconducting correlations trough the interfaces both the singlet $f_{s}$ and the triplet $\vec{f}_{t}$ pair correlations are spread out through the whole structure. As a result also the magnetization extends through the whole system, including the superconductor. The induced spin magnetization below $T_{c}$ is defined as

$$
\delta \vec{M}(x)=2 \mu_{B} \mathcal{N}_{f} T \sum_{n} \vec{g}_{t}\left(x, \epsilon_{n}\right),
$$

where $\mu_{B}$ is the Bohr magneton. In Fig. 2] we present the induced magnetization within the trilayer for parallel and
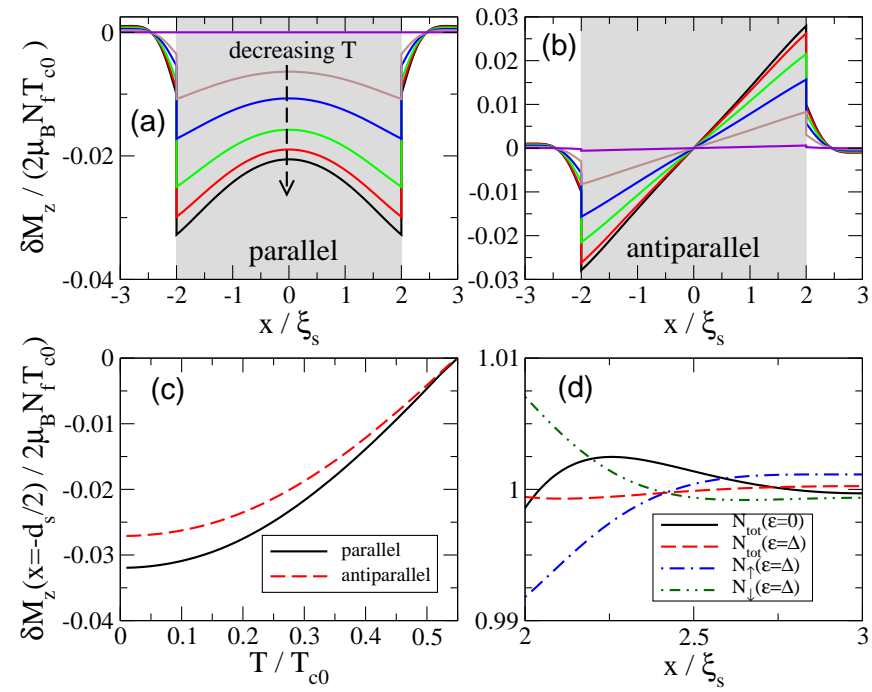

FIG. 2: Spatial dependence of the induced magnetization at various $T \in[0.05,0.55] T_{c 0}$ in steps of $0.1 T_{c 0}$ for (a) parallel $\left(\theta_{J}=0, \overrightarrow{J_{1}} \uparrow \uparrow \overrightarrow{J_{2}}\right)$ and (b) antiparallel $\left(\theta_{J}=\pi, \vec{J}_{1} \uparrow \downarrow \overrightarrow{J_{2}}\right)$ configurations. The $\mathrm{S}$ region is shaded. (c) Dependence of $\delta M_{z}$ on $T$ at the left interface (in $\mathrm{S}$ ) for both configurations. (d) Spatial dependence of the normalized LDOS in $\mathrm{F}_{2}$ at $\epsilon=0$ and at $\epsilon=\Delta(x=0)=1.16 T_{c 0}$ for the parallel configuration. Here, $d_{F 1}=d_{F 2}=\xi_{S}, d_{S}=4 \xi_{S}, J_{1}=J_{2}=20 T_{c 0}, \sigma_{F}=\sigma_{S}$, $D_{F}=D_{S}$, and both interfaces have $r_{b}=0.1$ and $\mathcal{T}=1$.

antiparallel orientations of the exchange fields. We see in (a)-(b) that $\delta \vec{M}$ extends into the superconductor over the distance $\sim \xi_{S}$, while decaying and oscillating on the magnetic scale $\xi_{J}=\sqrt{D_{F} / J}$ in the ferromagnets. There is a large increase in the magnitude of $\delta \vec{M}$ as the temperature is lowered well below $T_{c}$, see Fig. 2(c). The corresponding spatial variations of the LDOS [22] are shown in Fig. 2(d). The LDOS is spin split as due to the presence of triplet correlations. The order of magnitude of the LDOS modulations ( $1 \%$ effect) is in agreement with experiments [15].

The singlet order parameter is suppressed at the interface to the ferromagnets. At the same time, the singlet correlations leak into the ferromagnets. For parallel or antiparallel orientations of the exchange fields, triplet pairing correlations with zero spin projections are induced. For other orientations equal spin pairs are also induced. In Fig. 3(a)-(b) we show for perpendicular orientation the spatial dependence of the order parameter $\Delta(x)$ and of the correlation functions defined as 23.

$$
\begin{aligned}
& \Phi_{s}(x)=2 T \sum_{n>0} f_{s}\left(\epsilon_{n}, x\right), \\
& \vec{\Phi}_{t}(x)=T \sum_{n>0} \vec{f}_{t}\left(\epsilon_{n}, x\right) .
\end{aligned}
$$

Note that because $\vec{f}_{t}\left(-\epsilon_{n}, x\right)=-\vec{f}_{t}\left(\epsilon_{n}, x\right)$, the total sum over all frequencies in Eq. (7) would vanish. Since we assume an energy-independent pairing interaction there 

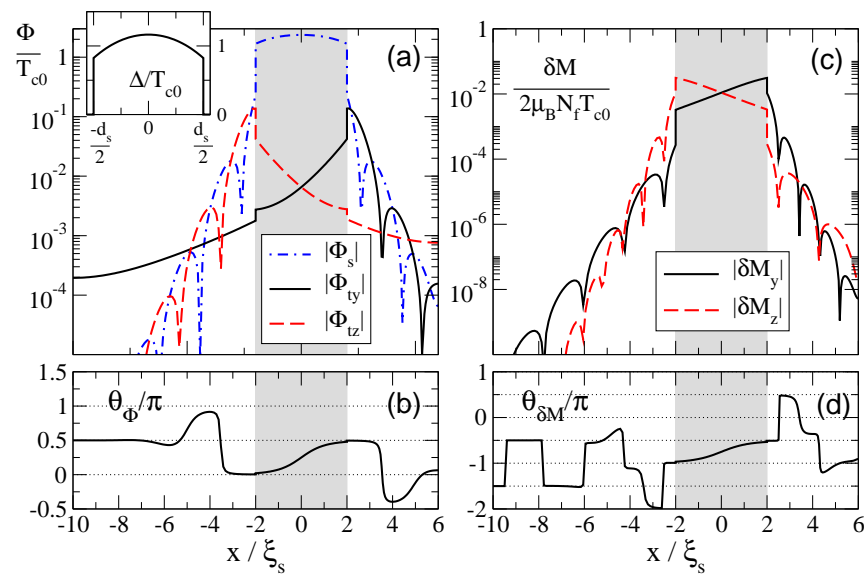

FIG. 3: Spatial dependence (a) of the singlet $\left(\Phi_{s}\right)$ and triplet $\left(\vec{\Phi}_{t}\right)$ pairing amplitudes, and (c) of the induced magnetization $\delta \vec{M}$ for perpendicular configuration $\left(\vec{J}_{1} \perp \vec{J}_{2}, \theta_{J}=\pi / 2\right)$. Here, $d_{F 1}=8 \xi_{S}, d_{F 2}=d_{S}=4 \xi_{S}$, and $T=0.1 T_{c 0}$. The other parameters are as in Fig. 2 The $\mathrm{S}$ region is shaded. (a) The singlet $\Phi_{s}(x)$ (dotted line) leaks into the $\mathrm{F}$ regions and oscillates and decays on a short length scale. The triplets $\Phi_{t y}(x)$ (full line) and $\Phi_{t z}(x)$ (dashed line) are short-range or long-range depending on their respective projections on $\overrightarrow{J_{1}}$ or $\vec{J}_{2}$. Inset: spatial dependence of $\Delta(x)$ in the $\mathrm{S}$ region. (c) There is an asymmetry in the oscillation period between $\delta M_{y}$ (full line) and $\delta M_{z}$ (dashed line) in both $\mathrm{F}$ regions. (b) and (d): The angles $\theta_{\Phi}$ and $\theta_{\delta M}$ relative to the $z$-axis, that quantify the directions of $\vec{\Phi}_{t}$ and $\delta \vec{M}$ in the $y$-z-plane.

is no triplet order parameter. The singlet component is purely real, while the triplet components are purely imaginary. The singlet component and the triplet component with zero spin projection on the local exchange field oscillate out of phase with respect to each other, and decay fast in the ferromagnets on the magnetic length scale $\xi_{J}$ [9, 24]. As can be seen in Fig. 3(a)-(b), there are long-range triplet pairing correlations in both ferromagnetic layers. The component $\Phi_{t y}$ is decaying slowly in $\mathrm{F}_{1}$ while $\Phi_{t z}$ is decaying slowly in $\mathrm{F}_{2}$. Relative to the local exchange fields, these components describe the equal spin pairing correlations that decay on the coherence length scale $\xi_{T}=\sqrt{D_{F} / 2 \pi T}$ but do not oscillate 1, 2]. It should be noted, however, that these components are already at the interfaces quite small since they are induced non-locally in one ferromagnet by diffusion from the other through the superconductor.

In Fig. 31 (c)-(d) we show the spatial dependence of the induced magnetization for perpendicular orientation of the exchange fields. Again, $\delta \vec{M}$ is spread out over the large length scale $\xi_{S}$ in the superconductor. But $\delta \vec{M}$ decays rapidly in the ferromagnet on the scale $\xi_{J}$, because the spin-vector Green function $\vec{g}_{t}$ is proportional to the fast decaying spin singlet component $f_{s}$ through the relation [5] $g_{s} \vec{g}_{t}=f_{s} \vec{f}_{t}$. As a further consequence of this relation, the oscillation periods are different for the
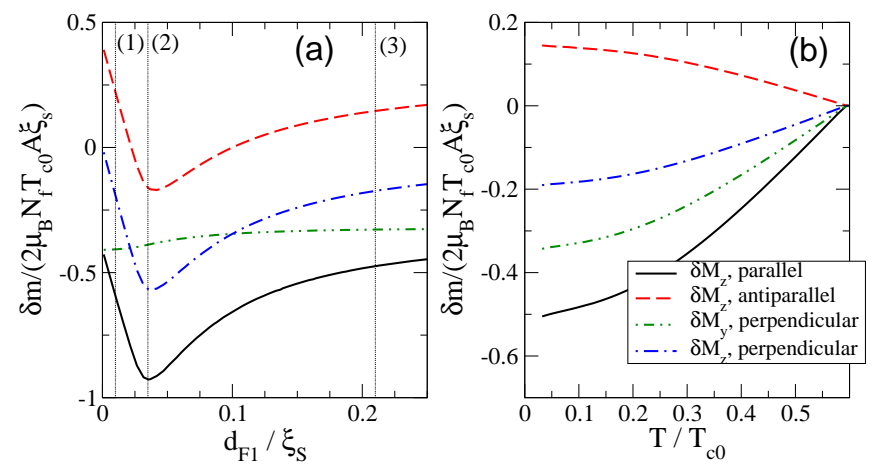

FIG. 4: (a) Change in magnetic moment $\delta \vec{m}$ of a trilayer as function of the layer thickness $d_{F 1}$, for $d_{S}=4 \xi_{S}, d_{F 2}=0.1 \xi_{S}$, $T=0.1 T_{c}$, and $J_{1}=J_{2}=20 T_{c}$. The other parameters are as in Fig. 2 (b) The temperature dependence of $\delta \vec{m}$ for parameters as in (a), but with $d_{F 1}=0.2 \xi_{S}$. The vertical lines in (a) are referred to in Fig. [5]

two components of $\delta \vec{M}$. For example, in $\mathrm{F}_{1}$ the oscillation period of $\delta M_{y}$ is twice that of $\delta M_{z}$. The reason is that $\delta M_{y}$ is determined by the product of the oscillating $f_{s}$ and the monotonic $f_{t y}$ in Fig. 3(a), while $\delta M_{z}$ is determined by the product of the oscillating $f_{s}$ and the oscillating $f_{t z}$. Note that deep in the ferromagnet (for $x<-5 \xi_{S}$ in $\mathrm{F}_{1}$ ), the magnetization change is mainly due to the long-range triplet correlations $f_{t y}$ and $\delta \vec{M}$ is therefore directed along the $y$-axis. But the magnitude of $\delta \vec{M}$ is exponentially small in this region. An observation of the two oscillation periods of the two components $\delta M_{z}$ and $\delta M_{y}$, reflecting the different behavior of the short-range oscillating $f_{t z}$ and the long-range monotonic $f_{t y}$, would be a smoking gun for long-range triplet components. Note that we have chosen a rather large value $J=20 T_{c 0}$ in order to clearly separate the length scales in the problem. Smaller values of $J$ might be more favorable in order to experimentally resolve this effect.

Let us further address the issue of how to experimentally find fingerprints of the triplet superconducting correlations. We have seen that the local magnetization change is rather small. But it is important to realize that the integrated effect,

$$
\delta \vec{m}=\mathcal{A} \int d x \delta \vec{M}(x),
$$

can be large. To illustrate this, we show in Fig. 4 the total magnetic moment of the trilayer, $\delta \vec{m}$, for various orientations of the exchange fields, both as a function of $d_{F 1}$ and of $T$. We suggest to exploit the largeness of the integrated effect by measuring the torque on the trilayer in a weak external magnetic field. We consider a field $\vec{B}$ in the $y$-z-plane, aligned at an angle $\varphi$ relative to the $z$-axis, as shown in Fig. प(b). Since the magnetic moment $\vec{m}$ of the trilayer is also in the $y$-z-plane, the torque $\vec{\tau}=\vec{m} \times \vec{B}$ is directed along the $x$-axis. We assume that the external field is very weak so that the moment can be 

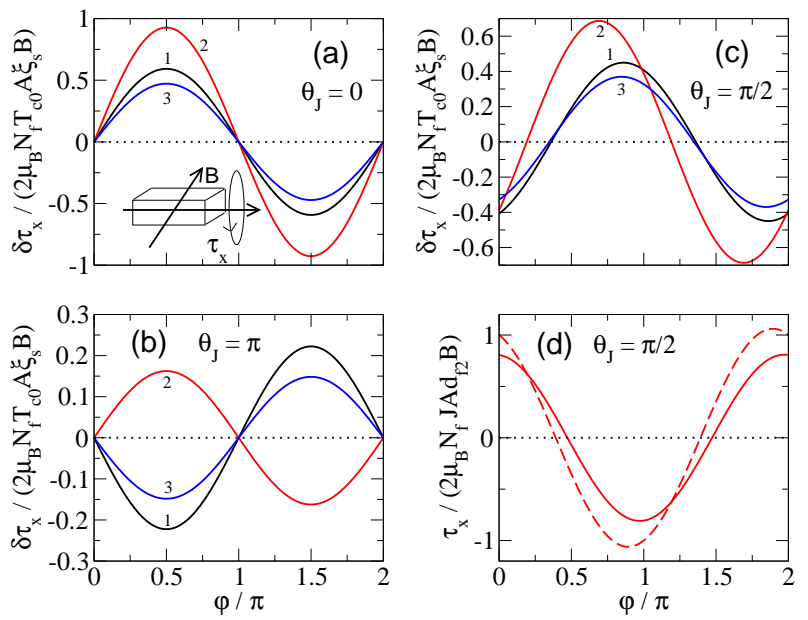

FIG. 5: (a)-(c) The change in the torque relative to that in the normal state for parallel $\left(\theta_{J}=0\right)$, antiparallel $\left(\theta_{J}=\pi\right)$, and perpendicular $\left(\theta_{J}=\pi / 2\right)$ orientations. The parameters are the same as in Fig. 4 with the three numbered curves corresponding to the three layer thicknesses $d_{F 1}=$ $\{0.01,0.035,0.21\} \xi_{S}$ (indicated by dashed vertical lines in Fig. 4). In (d) we compare the total torque in the normal state (dashed line) with the one in the superconducting state (solid line from curve 2 in (c)) within the itinerant ferromagnet model.

computed at $B=0$. In the normal state there is a torque due to the magnetic moment of the ferromagnetic layers. As the sample is cooled down through $T_{c}$, the magnetic moment changes, and consequently the torque is modified. This is illustrated for the parallel and antiparallel exchange field orientations in Fig. 5(a)-(b). In addition, when the exchange fields are not collinear, for example oriented perpendicular to each other as in Fig. 5 (c), the $\varphi$-dependence changes for asymmetric structures (here the layer thicknesses are different, $d_{F 1} \neq d_{F 2}$ ). The shift of the maxima in $\delta \tau_{x}(\varphi)$ when entering the superconducting state, as e.g. in curve 2 in Fig. 5 (c), can be negative or positive depending on the parameters of the trilayer.

In order to estimate the size of the change in the torque between the normal and superconducting state, we use the itinerant ferromagnet model. In this case the magnetization in the normal state is directly related to the local exchange fields in the two layers as $\vec{M}_{1 / 2}^{N}=2 \mu_{B} \mathcal{N}_{f} \vec{J}_{1 / 2}$. The corresponding magnetic moments are $\vec{m}_{1 / 2}^{N}=2 \mu_{B} \mathcal{N}_{f} \vec{J} \mathcal{A} d_{F 1 / 2}$. The magnetic moments in the normal and superconducting states are then $\vec{m}^{N}=\vec{m}_{1}^{N}+\vec{m}_{2}^{N}$ and $\vec{m}^{S}=\vec{m}^{N}+\delta \vec{m}$, respectively. In Fig. [5(d) we show how the torque is modified within this model. In particular, the equilibrium orientation $(\varphi$ for which $\vec{m} \| \vec{B}$ ) is different in the normal and superconducting states and the maximum in $\tau_{x}(\varphi)$ shifts to smaller $\varphi$ by $\sim 10 \%$. The magnitude of these effects increases when parameters are such that the spin screening is more efficient (e.g. smaller $J$ or smaller $d_{F 1 / 2}$ ).
In summary, we have shown that superconducting triplet correlations in FSF trilayers are much easier detected by measurements of global properties of the sample rather than by local probes. As an example we have studied the induced magnetic moment in the superconducting state. The induced magnetic moment is a direct consequence of the presence of triplet pairing correlations in the trilayer. In an external magnetic field, the torque on the trilayer is modified in the superconducting state. We have shown that this effect can be more than $10 \%$ for exchange fields as large as $|\vec{J}| \sim 20 T_{c 0}$. The proposed effect can be used to experimentally determine the presence of triplet correlations.

We acknowledge financial support from the EC under the spintronics network RTN2-2001-00440 (T.L.) and the Deutsche Forschungsgemeinschaft within the Center for Functional Nanostructures (T.C. and M.E.).

[1] F. S. Bergeret, A. F. Volkov, and K. B. Efetov, Phys. Rev. Lett. 86, 4096 (2001); cond-mat/0506047 (2005).

[2] A. Kadigrobov, R. I. Shekhter, and M. Jonson, Europhys. Lett. 54, 394 (2001).

[3] M. Eschrig et al., Phys. Rev. Lett. 90, 137003 (2003).

[4] A.I. Buzdin, cond-mat/0505583 (2005).

[5] T. Champel and M. Eschrig, Phys. Rev. B 71, 220506(R) (2005); Phys. Rev. B (to appear, 2005), cond-mat/0504198

[6] V. N. Krivoruchko and E. A. Koshina, Phys. Rev. B 66, 014521 (2002).

[7] F. S. Bergeret, A. F. Volkov, and K. B. Efetov, Phys. Rev. B 69, 174504 (2004).

[8] Y. V. Fominov, A. A. Golubov, and M. Y. Kupriyanov, JETP Letters 77, 510 (2003).

[9] Z. Radović et al., Phys. Rev. B 44, 759 (1991).

[10] R. Fazio and C. Lucheroni, Europhys. Lett. 45, 707 (1999).

[11] K. Halterman and O.T. Valls, Phys. Rev. B 65, 14509 (2001).

[12] A. F. Volkov, F. S. Bergeret, and K. B. Efetov, Phys. Rev. Lett. 90, 117006 (2003).

[13] J. Stahn et al., et al., Phys. Rev. B 71, 140509(R) (2005).

[14] V. V. Ryazanov et al., Phys. Rev. Lett. 86, 2427 (2001).

[15] T. Kontos et al., Phys. Rev. Lett. 86, 304 (2001).

[16] K. D. Usadel, Phys. Rev. Lett. 25, 507 (1970).

[17] J. A. X. Alexander et al., Phys. Rev. B 31, 5811 (1985).

[18] L.N. Bulaevskii et al., Adv. Phys. 34, 175 (1985).

[19] Y. V. Nazarov, Superlatt. Microstr. 25, 1221 (1999).

[20] We assume here for simplicity that all conductance channels contribute with equal weight.

[21] M. Eschrig et al., Adv. in Sol. State Phys. 44, 533 (2004).

[22] The LDOS is calculated from the real energy version of Eq. (2). The spin resolved (along $\hat{n}$ ) LDOS is then defined as $N_{\hat{n}}=-\left(\mathcal{N}_{f} / \pi\right)\left[g_{s}+\hat{n} \cdot \vec{g}_{t}\right]$, while the total LDOS is the sum of spin up and spin down contributions.

[23] These sums converge except for Eq. (6) in the S region, for which we used a frequency sum cut-off of $10 T_{c 0}$.

[24] A.I. Buzdin, L.N. Bulaevskii, and S.V. Panyukov, Pis'ma Zh. Eksp. Teor. Fiz. 35, 147 (1982) [JETP Lett. 35, 178 (1982)]. 